\title{
Aetiology and risk factors for neonatal sepsis at the Lagos University Teaching Hospital, Idi-Araba, Lagos, Nigeria
}

\author{
E O Shobowale, ${ }^{1}$ MBBS, MSc, FMC Path; F T Ogunsola, ${ }^{2}$ MBBS, MSc, PhD, FMC Path, FWACP; \\ O O Oduyebo, ${ }^{2}$ MBBS, MSc, FMC Path, FWACP; V I Ezeaka, ${ }^{3}$ MBBS, FMC Paed, FWACP \\ ${ }^{1}$ Department of Medical Microbiology and Parasitology, Babcock University Teaching Hospital, Ogun, Nigeria \\ ${ }^{2}$ Department of Medical Microbiology and Parasitology, Lagos University Teaching Hospital, Lagos, Nigeria \\ ${ }^{3}$ Department of Paediatrics, Lagos University Teaching Hospital, Lagos, Nigeria
}

Corresponding author: E O Shobowale (shoekineh@gmail.com)

\begin{abstract}
Background. Neonatal sepsis is a significant cause of morbidity and mortality in developing countries, accounting for a large proportion of neonatal deaths annually. Every year, 4 million neonates die, and one-third of these deaths is attributed directly to neonatal sepsis.

Objectives. To determine the prevalence of neonatal sepsis, characterise and identify causative organisms and identify possible risk factors. Specific objectives were to determine the aetiological agents responsible for neonatal sepsis at Lagos University Teaching Hospital and also to identify the risk factors responsible for the development of neonatal sepsis.

Methods. Venous blood pairs were collected from clinically septic admitted neonates and inoculated into BACTEC Peds Plus (BD, USA) bottles aerobically in the BACTEC 9050 system. Organisms were identified using the Microbact 12A/E system and biochemicals. A structured questionnaire was used to collect data for risk factors, which were analysed with the SPSS version 17.

Results. Of 250 neonates who were sampled, 85 (34\%) had pathogens recovered from their bloodstream, with Klebsiella pneumoniae the predominant organism. Risk factors for sepsis were being delivered outside the hospital $(p=0.01)$, and by frequent changes in antibiotics $(p=0.00)$. Conclusion. The burden of neonatal sepsis is still high in our environment as evidenced by our isolation rate of $34 \%$. A concerted effort needs to be made to reduce this.
\end{abstract}

S Afr J Child Health 2016;10(3):147-150. DOI:10.7196/SAJCH.2016.v10i3.965

It is estimated that $20 \%$ of all neonates will develop sepsis, and $~ 1 \%$ of them will die of sepsis-related causes. In addition, neonatal deaths account for $43 \%$ of all deaths among children under 5 years. ${ }^{[1]}$ In developing countries such as Nigeria, which share $99 \%$ of the estimated 4 million neonatal deaths annually, neonatal mortality resulting from neonatal sepsis is estimated to be $\sim 34 / 1000$ live births, while in developed countries it is $\sim 5 / 1000 .^{[1,2]}$ Neonatal morbidity and mortality are major public health challenges in our local environment, with a huge percentage of deaths in the neonatal period attributable to sepsis. It is estimated that $98.5 \%$ of neonatal mortality occurs in developing countries, with neonatal sepsis directly responsible for $26 \%$ of neonatal deaths ${ }^{[1]}$ Neonatal sepsis in itself is potentially treatable and preventable, yet despite considerable advances and improvements in the survival rate of newborns in developed countries, there has not been a concomitant improvement in outcomes recorded in developing countries. ${ }^{[1]}$

Neonatal sepsis is classically divided into early- and late-onset sepsis. Both are associated with different distributions of pathogens. Early-onset sepsis occurs in the first 7 days of life and is generally acquired from pathogens in the maternal genital tract, whereas late-onset sepsis, which occurs after the 7th day of life, has its origin either in the community or in the healthcare environment. ${ }^{[3]}$ The aetiology of neonatal sepsis varies geographically, with different regions reporting a plethora of prevalent pathogens. Organisms such as Escherichia coli, Klebsiella pneumoniae, Staphylococcus aureus, coagulase-negative staphylococci, Enterococcus spp., Proteus spp., and Pseudomonas aeruginosa have been reported. ${ }^{[3]}$

The objective of the current study was to ascertain and identify the local risk factors responsible for bloodstream infections among neonates at the Lagos University Teaching Hospital (LUTH), and aid in generating baseline data for the prevalent micro-organisms responsible for neonatal sepsis in our institution, alongside susceptibility and resistance patterns. This also helped to provide relevant information on the practices in our neonatal units that are predisposed to sepsis. These data were used by clinicians to predict susceptibility to antimicrobial agents on an empirical basis while awaiting susceptibility results and the identification of pathogens.

\section{Methods}

The study was carried out at the neonatal unit of the LUTH, Idi-Araba, Lagos, Nigeria. The hospital is a 761-bed facility located in an urban cosmopolitan setting. The neonatal unit of the hospital has a total bed space of 73 and is divided into four wards: neonatal unit (NNU), and wards D1, CHER and E4. The sample size was calculated, using the average isolation rate of aerobic bacteria from manual blood culture systems of $\sim 20 \%{ }^{[4]}$ A sample of 250 neonates was determined. ${ }^{[5]}$ The study included neonates delivered within or outside the hospital, who had signs and symptoms of sepsis. Blood samples for culture were collected within 48 hours of admission. These samples included nosocomial sepsis and maternal acquisition. All the neonates recruited into the study were admitted to the hospital and they presented with clinical signs and symptoms of sepsis, including fever, hypothermia, lethargy, bulging fontanelle, irritability, seizures, apnoea and failure to thrive in the first 28 days of life. Sampling was limited to blood cultures for the study, as lumbar punctures for cerebrospinal fluid analysis were performed by the managing team only when indicated. Neonates without clinical signs and symptoms of sepsis and those whose parents withheld consent were excluded from the study.

Two venous blood samples were taken via phlebotomy from the antecubital fossa, forearm or hands of the neonates aseptically, applying universal precautions. The volume of blood withdrawn was $1-3 \mathrm{~mL}$. Blood obtained from each neonate was aseptically 
Table 1. Demographic characteristics of neonates investigated for sepsis at LUTH

Characteristics $\quad n(\%)(N=250)$

\section{Gender}

Male

$118(47.2)$

Female

$132(52.8)$

Age (days)

$\begin{array}{ll}0-6 & 151(60.4) \\ 7-28 & 99(39.6)\end{array}$

Socioeconomic status ${ }^{*}$

$\begin{array}{ll}\text { High } & 18(7.0) \\ \text { Middle } & 50(19.8) \\ \text { Low } & 182(72.3)\end{array}$

${ }^{*}$ High socioeconomic status: combined income of >USD5 000 per month; middle socioeconomic status: combined income of USD1 000 - USD5 000 per month; low socioeconomic status: combined income of $<$ USD1 000 per month.

dispensed into the BACTEC Peds Plus (BD, USA) aerobic blood culture bottle, placed in the BACTEC 9050 machine and incubated for a maximum of 5 days. Bottles flagged as positive by the system were removed, Gram stained and sub-cultured onto appropriate media such as blood, chocolate (for Grampositive organisms) and MacConkey agar (for Gram-negative organisms) and identified using biochemical tests. All isolates were tested for antimicrobial susceptibility with the modified Kirby-Bauer method.

A structured questionnaire was given to patients' relatives/caregivers to complete in order to identify and assess risk factors. Data obtained from the questionnaires were analysed with Epi Info 3.5.1 software version 2008 (CDC, USA) and SPSS version 21.0 (IBM Corp., USA) by cross-tabulation of risk factors and univariate/multivariate analysis with data from the laboratory results. Analysis was done with the $\chi^{2}, 95 \%$ confidence interval (CI) and odds ratio (OR). Significance set at $p<0.05$.

Approval was obtained from the Ethics and Research Committee of the LUTH. Informed consent was obtained before the filling in of questionnaires.

\section{Results}

There was a predominance of female $(n=132,52.8 \%)$ compared with male neonates $(n=118,47.2 \%)$, giving a male to female ratio of $1: 1.12$. The mean age of the neonates was 9.1 days, with most of them $<7$ days $(n=151,60.4 \%)$. The majority of the neonates $(n=182,72.2 \%)$ were delivered to parents in the lower socioeconomic strata, with $50(19.7 \%)$ in the mid-strata and the remaining $18(17 \%)$ in the higher socioeconomic strata (Table 1 ).
Table 2. Aetiological agents stratified by age of onset of sepsis

\begin{tabular}{llll}
\hline Organism & $\boldsymbol{n}(\%)$ & Early onset, $\boldsymbol{n}(\%)$ & Late onset, $\boldsymbol{n}(\%)$ \\
\hline Acinetobacter baumanii & $3(3.5)$ & $2(67.0)$ & $1(33.0)$ \\
Acinetobacter iwoffii & $1(1.2)$ & $0(0.0)$ & $1(100)$ \\
Burkholderia cepacia & $6(7.0)$ & $4(67.0)$ & $2(33.0)$ \\
Candida albicans & $1(1.2)$ & $0(0.0)$ & $1(100)$ \\
CONS & $10(11.8)$ & $6(60.0)$ & $4(40.0)$ \\
Enterococcus spp. & $6(7.0)$ & $3(50.0)$ & $3(50.0)$ \\
Klebsiella oxytoca & $3(3.5)$ & $3(100)$ & $0(0.0)$ \\
K. pneumoniae & $31(36.5)$ & $19(61.3)$ & $12(38.7)$ \\
Proteus vulgaris & $4(4.7)$ & $3(75.0)$ & $1(25.0)$ \\
S. aureus & $16(18.8)$ & $9(56.3)$ & $7(43.7)$ \\
Serratia rubidaea & $4(4.7)$ & $1(25.0)$ & $3(75.0)$ \\
Total & $85(100)$ & $50(100)$ & $35(100)$ \\
CONS = coagulase-negative staphylococcus. & &
\end{tabular}

Table 3. Significant risk factors for sepsis in neonates at LUTH

\begin{tabular}{|c|c|c|c|c|c|}
\hline Risk factor & $\begin{array}{l}\text { Organism in } \\
\text { bloodstream, } \\
n(\%)\end{array}$ & $\begin{array}{l}\text { No organism in } \\
\text { bloodstream, } \\
n(\%)\end{array}$ & $p$-value & OR & 95\% CI \\
\hline Birth status & & & 0.1475 & 0.67 & $0.39-1.15$ \\
\hline Inborn & $30(35.3)$ & $74(44.8)$ & & & \\
\hline Outborn & $55(64.7)$ & $91(55.2)$ & & & \\
\hline Change in antibiotics & & & 0.0001 & 0.33 & $0.19-0.58$ \\
\hline Yes & $46(54.1)$ & $129(78.2)$ & & & \\
\hline No & 39 (45.9) & $36(21.8)$ & & & \\
\hline $\begin{array}{l}\text { Number of switches in } \\
\text { antibiotics }\end{array}$ & & & 0.0024 & 0.41 & $0.23-0.73$ \\
\hline 0 & $40(47.1)$ & $132(80.0)$ & & & \\
\hline$>1$ & 45 (52.9) & $33(20.0)$ & & & \\
\hline
\end{tabular}

With regard to aetiology, K. pneumoniae $(n=31)$ was the most frequently recovered pathogen in both early-onset $(n=19)$ and late-onset $(n=12)$ sepsis. It was followed by S. aureus ( $n=16,9$ early onset and 7 late onset). Coagulase-negative staphylococci were found in 10 neonates, with 6 cases in early-onset and 4 in late-onset sepsis. This was followed by Enterococcus spp., which accounted for 6 cases spread evenly between early- and late-onset variants. There was also one case of candidaemia in early-onset sepsis (Table 2).

The following were found to be risk factors for sepsis: patients born outside a tertiary hospital tended to be more at risk ( $p=0.15$, OR 0.67 , CI $0.39-1.15)$. A change in antibiotic therapy was the second risk factor observed, as those who had any change in antimicrobials were less likely to acquire sepsis $(p=0.0001$, OR $0.33,95 \%$ CI 0.19 - 0.58). Also, one or more switches in antibiotics was another identified factor $(p=0.0024$, OR 0.41 , CI $0.23-0.73$ ) (Table 3).
The single statistically significant risk factor for early- v. late-onset sepsis was instrumentassisted delivery ( $p=0.02$, OR $3.26,95 \%$ CI 1.19 - 8.97). Babies who had instrumentassisted delivery were 3.26 times more likely to have late-onset sepsis than other neonates with suspected sepsis (Table 4).

\section{Discussion}

The findings from our study revealed that Gram-negative organisms were predominant, as they accounted for $61.1 \%$ of organisms recovered, with K. pneumoniae the most frequent Gram-negative pathogen. This finding is in keeping with results obtained by other researchers in previous studies where K. pneumoniae alone was responsible for $30 \%$ of cases of neonatal sepsis. ${ }^{[5-7]}$ Klebsiella spp. were also the most frequent cause of sepsis at neonatal intensive care units (NICUs) in other resource-constrained settings. ${ }^{[8]}$

The majority of bloodstream infections due to K. pneumoniae were of early onset 
Table 4. Significant risk factor identified for early- and late-onset of sepsis

\begin{tabular}{llllll}
\hline Risk factor & Early onset, $\boldsymbol{n}(\%)$ & Late onset, $\boldsymbol{n}(\%)$ & $\boldsymbol{p}$-value & OR & $\mathbf{9 5 \%} \mathbf{C I}$ \\
\hline $\begin{array}{l}\text { Instrument- } \\
\text { assisted delivery }\end{array}$ & & & 0.02 & 3.26 & $1.19-8.97$ \\
No & $41(83.7)$ & $22(61.1)$ & & & \\
Yes & $8(16.3)$ & $14(38.9)$ & & &
\end{tabular}

$(n=19 / 31,61.3 \%)$. This is also in line with some previous studies which show this organism to be one of the common causes of early-onset sepsis. ${ }^{[4,9-16]}$ A study from Bangladesh also revealed that Gramnegative organisms were responsible for $73 \%$ of episodes of neonatal sepsis, with E. coli identified as the most common cause (30\%), followed by Klebsiella spp. $(23 \%){ }^{[12]}$ This highlights the predominance of Gram-negative bacteria in the aetiology of neonatal sepsis; clinicians need to bear this in mind when instituting antimicrobial therapy. However, this pattern is at variance with another study which demonstrated a preponderance of Gram-positive pathogens, highlighting the unique micro-ecological niche of different healthcare facilities. ${ }^{[7]}$ The import of this is that therapeutic regimens should cover both groups of pathogens pending the definitive identification and susceptibility profiles of the agents responsible for sepsis.

A similar pattern was also seen in a study from this centre 11 years ago, which reported $K$. pneumoniae as the predominant pathogen implicated in neonatal sepsis. The findings from this research show that our microecology, with regard to the predominant pathogens, has not changed, and also indicates that a case needs to be put forward for stringent infection control measures as well as antimicrobial stewardship. S. aureus was the next most common pathogen; this is in line with findings from previous studies that describe it as a frequent cause of sepsis in neonates. ${ }^{[13]}$ However, the isolation rate of $18.8 \%$ for $S$. aureus from this study is less than that obtained by workers at Illorin in Nigeria. ${ }^{[14]}$

It is possible that some of these pathogens may have been transmitted from healthcare personnel or hospital equipment: therefore, continuous and regular training of neonatal unit staff on proper handwashing techniques before and after touching babies will help to reduce the transmission of pathogens in the NICU setting, as this pathogen was recovered from the hands of some healthcare workers.

The following risk factors for neonatal sepsis were identified in the course of the study: delivery outside this teaching hospital, change in antibiotics from the initial regimen and frequent changes in antibiotics. The reasons for these findings could be related to poor levels of antenatal, intrapartum and postnatal care, insufficient level of knowledge on the part of attending physicians in those facilities of appropriate antimicrobial use, and delayed detection of neonates at risk of sepsis. Babies delivered outside this tertiary hospital were not more likely to develop sepsis when compared with neonates born at our hospital. However, in order to reduce sepsis rates among our neonates, it is imperative that physicians in our referral centres be trained and retrained on the potential signs of sepsis and that they know what broad-spectrum antimicrobial agents they need to administer based on local susceptibility patterns. Our results also showed that early-onset sepsis was more common than late-onset sepsis, which is compatible with findings from previous studies. ${ }^{[17]}$

A previous study identified the following risk factors for sepsis: lack of antenatal care, maternal colonisation with group B streptococci, preterm labour, low- and very low birth weight, instrumentation and invasive procedures, handling by healthcare workers, prolonged use of antibiotics and resuscitation at birth ${ }^{[18]}$ Early-onset sepsis was more common than late-onset in our study. This is keeping with previous data that demonstrate a marked propensity for sepsis in the first 7 days of life, given that this is the stage at which neonates are most susceptible to infectious agents.

The performance of invasive procedures, including mechanical ventilation and instrument-assisted delivery, was more common in the early-onset cases owing to this being the stage at which infants are most vulnerable to illnesses and require some form of intervention. It is essential that policies are formulated and strengthened to guide clinicians in the application of safe interventional procedures geared towards reducing the risk of sepsis.

The relatively high rate of early-onset sepsis detected in this study provides a reservoir of infectious neonates who pose a considerable risk of nosocomial transmission to other neonates. Numerous studies have reported that the most common pathogens isolated in early-onset sepsis include: group B streptococci (which was not found in this study), S. aureus, E. coli (also not seen), Klebsiella spp. and Listeria monocytogenes. ${ }^{[8,19]}$ The data from this study show that the pattern of bacterial isolates in our local centre differ to previously published data and therefore the use of different antibiotic guidelines based on local susceptibility data will be warranted. There is therefore the need to formulate local guidelines that will aid in the rapid identification of atrisk neonates, especially those who have developed sepsis, and develop treatment modules that will help to reduce mortality in our centre. This study has outlined the local risk factors that impact on sepsis in our environment. The identification and use of these factors will help to form a framework for those who may be at risk of acquiring sepsis and help improve outcome in those who are identified early at being at risk of sepsis.

\section{Study limitations}

There were challenges in obtaining additional data on risk factors for sepsis, as some of these neonates were referred from other healthcare facilities to the study site and did not come with information on possible risk factors.

\section{Conclusion}

Our predominant pathogens were Klebsiella spp. and $S$. aureus. The burden of neonatal sepsis is still high in our environment, as evidenced by our isolation rate of $34 \%$. The risk factors identified included: delivery outside a tertiary hospital, changes in antibiotic therapy and instrument-assisted delivery. A concerted effort needs to be made to reduce this scenario.

\section{Recommendations}

We recommend that babies presenting with respiratory distress and/or fever are screened immediately for bacterial sepsis. Infection control protocols also need to be instituted and strengthened in both our referral centres and teaching hospitals, with regular neonatal sepsis surveillance.

\section{References}

1. Wu JH, Chen CY, Tsao PN, Hsieh WS, Chou HC Neonatal sepsis: A 6-year analysis in a neonatal care unit in Taiwan, Pediatr Neonatol 2009;50(3):88 95. DOI:10.1016/S1875-9572(09)60042-5

2. Black RE, Cousens S, Johnson HL, et al., Global, regional, and national causes of child mortality in 2008: A systematic analysis. Lancet 2010;375(9730):1969-1987. DOI:10.1016/S01406736(10)60549-1

3. Stoll BJ, Hansen NI, Sanchez PJ, et al. Early onset neonatal sepsis: The burden of group B Streptococcal and E coli disease continues. Pediatrics 2011;127(5):817-826. DOI:10.1542/ peds.2010-2217

4. Iroegbu KC, Elegba YO, Babaniyi IB Bacteriological profile of neonatal septicemia in a tertiary hospital in Nigeria. Afr Health Sci 2006;6(3):151-154

5. Kish L. Survey Sampling. New York: John Wiley, 1965:49-50.

6. Zaidi AK, Hushus WK, Thower D, et al. Hospitalacquired neonatal infections in developing countries. Lancet 2005;365(9465):1175-1183. DOI:10.1016/S0140-6736(05)71881-X 
7. Ghotaslou R, GhorashiZ, Nahaei MR. Klebsiella pneumoniae in neonatal sepsis: A 3-year-study in the pediatric hospital of Tabriz, Iran. Jpn J Infect Dis 2007;60(2-3):126-128

8. Waters D, Jawad I, Ahmad A, et al. Aetiology of community-acquired neonatal sepsis in low and middle income countries. J Glob Health $2011 ; 1(2): 154-170$.

9. National Neonatology Forum NNPD Network. National Neonatal-perinatal Database: Report for 2002-2003. New Delhi: National Neonatology Forum NNPD Network, 2005.

10. Adejuyigbe EA, Adeodu OO, Ako-Nai KA, Taiwo O, Owa JA. Septicemia in high-risk neonates at a teaching hospital in Ile-ife, Nigeria. East Afr Med J 2001;78(10):540-543.

11. Orrett FA, Shurland SM. Neonatal sepsis and mortality in a regional hospital in Trinidad: Aetiology and risk factors. Ann Trop Paediatr 2001;21(1):20-25.

12. Awaisu A, Sulaiman SA, Ibrahim MI, Saad A. Antibiotics utilization and outcomes of neonatal sepsis among patients admitted to a university teaching hospital in Malaysia. Eastern J Med 2007;12:6-14.
13. Zaidi AK, Thaver D, Ali SA, Khan TA. Pathogens associated with sepsis in newborns and young infants in developing countries. Paediatr Infect Dis J 2009;28(1):S10-S18. DOI:10.1097/INF.0b013e3181958769

14. Mokuolu AO, Jiya N, Adesiyun OO. Neonatal septicemia in Ilorin: Bacterial pathogens and antibiotic sensitivity pattern. Afr J Med Sci 2002;31(2):127-130.

15. Aftab R, Iqbal I. Bacteriological agents of neonatal sepsis in NICU at Nishtar Hospital Multan. J Coll Physicians Surg Pak 2006;16(3):216-219.

16. Polin RA, Committee on Fetus and Newborn. Managements of neonates with suspected or proven early-onset neonatal sepsis. Pediatrics 2012;129(5):10061015. DOI:10.1542/peds.2012-0541

17. Movahedian AH, Moniri R, Mosayebi Z. Bacterial culture of neonatal sepsis. Iranian J Publ Health 2006;35(4):84-89.

18. Cohen-Wolkowiez M, Moran C, Benjamin DK, et al. Early and late onset sepsis in late preterm infants. Pediat Inf Dis J 2009;28(12):1052-1056.

19. Gandhi S, Ranjan KP, Ranjan N, Sapre N, Masani M. Incidence of neonatal sepsis in tertiary care hospital: An overview. Int J Med Sci Public Health 2013;2(3):548-552. DOI:10.5455/ijmsph.2013.090320131 